\title{
Overlap of Musical and Linguistic Syntax Processing: Intracranial ERP Evidence
}

\author{
D. Sammler, ${ }^{a, b}$ S. Koelsch,,${ }^{a, c}$ T. Ball, ${ }^{d, e}$ A. Brandt, ${ }^{d}$ C. E. Elger,${ }^{f}$ \\ A. D. Friederici, ${ }^{b}$ M. Grigutsch, ${ }^{b}$ H.-J. Huppertz, ${ }^{g}$ \\ T. R. Knösche, ${ }^{h}$ J. Wellmer, ${ }^{f}$ G. Widman, ${ }^{f}$ \\ and $A$. Schulze-Bonhage ${ }^{d, e}$
}

aMax Planck Institute for Human Cognitive and Brain Sciences, Independent Junior Research Group "Neurocognition of Music," Leipzig, Germany

${ }^{b}$ Max Planck Institute for Human Cognitive and Brain Sciences, Department of Neuropsychology, Leipzig, Germany

${ }^{c}$ Department of Psychology, University of Sussex, Brighton, United Kingdom

${ }^{d}$ Epilepsy Center, Neurocenter, University Hospital of Freiburg,

Freiburg im Breisgau, Germany

${ }^{e}$ Bernstein Center for Computational Neuroscience (BCCN), University of Freiburg, Freiburg im Breisgau, Germany

${ }^{f}$ Clinic of Epileptology, University Hospital of Bonn, Bonn, Germany

${ }^{g}$ Sreiss Epilepsy Centre, Zurich, Srwitzerland

${ }^{h}$ Max Planck Institute for Human Cognitive and Brain Sciences, Workgroup Cortical Networks and Cognitive Functions, Leipzig, Germany

\begin{abstract}
The present study investigated the co-localization of musical and linguistic syntax processing in the human brain. EEGs were recorded from subdural electrodes placed on the left and right perisylvian cortex. The neural generators of the early potentials elicited by syntactic errors in music and language were localized by means of distributed source modeling and compared within subjects. The combined results indicated a partial overlap of the sources within the bilateral superior temporal gyrus, and, to a lesser extent, in the left inferior frontal gyrus, qualifying these areas as shared anatomic substrates of early syntactic error detection in music and language.
\end{abstract}

Key words: music; language; syntax; subdural; event-related potential (ERP); ERAN; ELAN

\section{Introduction}

Both music and language are highly structured systems in which discrete elements are arranged according to a set of principles that may be denoted as syntax. Prevailing neurocognitive theories ${ }^{1,2}$ state that syntactic processing

Address for correspondence: Daniela Sammler, Max Planck Institute for Human Cognitive and Brain Sciences, Stephanstrasse 1a, 04103 Leipzig, Germany. Voice: +49 (0)341 9940 2467; fax: +49 (0)341 9940 113.sammler@cbs.mpg.de in both domains may rely on shared cognitive resources (although the syntactic representations per se may differ between domains). Intense investigation yielded ample evidence for this assumption: Surface event-related potential (ERP) studies showed similar early negativities (although with slightly different hemispheric weighting) during the processing of syntactic irregularities in either domain: The early left anterior negativity (ELAN) is elicited by word-category violations in sentences, ${ }^{3}$ and the early right anterior negativity (ERAN) is evoked by

The Neurosciences and Music III: Disorders and Plasticity: Ann. N.Y. Acad. Sci. 1169: 494-498 (2009).

doi: 10.1111/i.1749-6632.2009.04792.x @ 2009 New York Academy of Sciences. 
unexpected chords in harmonic progressions. ${ }^{4}$ Both negativities emerge about $200 \mathrm{~ms}$ after stimulus onset, exhibit an anterior scalp distribution, and are elicited by the violation of an expected structure, suggesting that they may reflect analogous neural mechanisms underlying early structure-building in music and language. Furthermore, it has been demonstrated that syntax processing interacts between both domains, ${ }^{5,6}$ that musical training enhances syntactic negativities in language, ${ }^{7}$ and that agrammatic disorders in language are associated with parallel deficits in music-syntactic processing. ${ }^{8,9}$ Overall, these data suggest a functional link between musical and linguistic syntax processing.

The present study set out to test how this functional overlap maps onto neural architecture. Likely candidates for shared resources are the bilateral inferior frontal (IFG) and superior temporal gyrus (STG), which have been previously associated with the generation of the ELAN ${ }^{10,11}$ and the ERAN. ${ }^{1,12}$ To further investigate the co-localization of musical and linguistic syntax processing in these areas, an intracranial ERP study was conducted, capitalizing on the excellent temporal and spatial resolution of such data to study the localization of transient ERP effects. Nine patients undergoing invasive EEG monitoring during evaluation for epilepsy were tested in a language comprehension $^{3}$ and a chord sequence paradigm. ${ }^{4}$ The electrocortical equivalents of the ELAN and ERAN were recorded from subdural electrodes placed within perisylvian brain regions in the left or right hemisphere, and subjected to distributed source modeling in order to localize and compare their neural generators. It was hypothesized that:

1. Syntactic violations in language and in music would elicit early negativities with a peak latency around $200 \mathrm{~ms}$.

2. The neural generators of these negativities would be located at identical or very similar coordinates within inferior frontal and superior temporal brain areas.

\section{Methods}

\section{Participants}

The study was conducted with nine righthanded patients (mean age: 31.6 years; six men and three women) undergoing presurgical evaluation of pharmaco-resistant epilepsy (mean duration of epilepsy: 19.6 years). All participants were German native speakers, and none of them was a professional musician.

\section{Stimuli}

In the language experiment, ${ }^{3}$ patients listened to 132 correct, 132 incorrect, and 66 filler sentences in the German language. Correct sentences contained a noun phrase $[\mathrm{Np}]$, an auxiliary [Aux], and a past participle [Pp] (e.g., The secret $_{[\mathrm{Np}]}$ was $_{[\mathrm{Aux}]}$ whispered $\left._{[\mathrm{Pp}]}.\right)$. In incorrect sentences, a preposition $[\mathrm{P}]$ was inserted before the $[\mathrm{Pp}]$ (literally translated, e.g., The $\left.\operatorname{plan}_{[\mathrm{Np}}\right]$ was $_{[\mathrm{Aux}]}$ in $_{\text {-the }}[\mathrm{P}]$ whispered $_{[\mathrm{Pp}]}$.). Since German grammar demands a $[\mathrm{P}]$ to be followed by a $[\mathrm{Np}]$, the immediate succession of a $[\mathrm{Pp}]$ represents a word-category violation. Correct filler sentences contained a correct succession of $[\mathrm{P}]$ and $[\mathrm{Np}]$ (e.g., The name ${ }_{[\mathrm{Np}]}$ was $_{[\mathrm{Aux}]}$ in-the $[\mathrm{P}]$ cranny $_{[\mathrm{Np}]}$ whispered $_{[\mathrm{Pp}]}$.).

In the music experiment, ${ }^{4}$ patients were presented with 144 regular and 144 irregular six-chord sequences. The first five chord functions of both sequence types were identical (dominant-tonic-subdominantsubdominant-dominant). Regular sequences ended on a highly expected tonic chord, whereas irregular sequences ended on the lessexpected major chord built on the lowered second degree.

Participants were not informed about the syntactic violations, but paid attention to infrequent changes of the speaker's voice or the musical instrument.

\section{Data Acquisition and Analysis}

The EEG was recorded from subdural grid electrodes placed on left (six patients) 

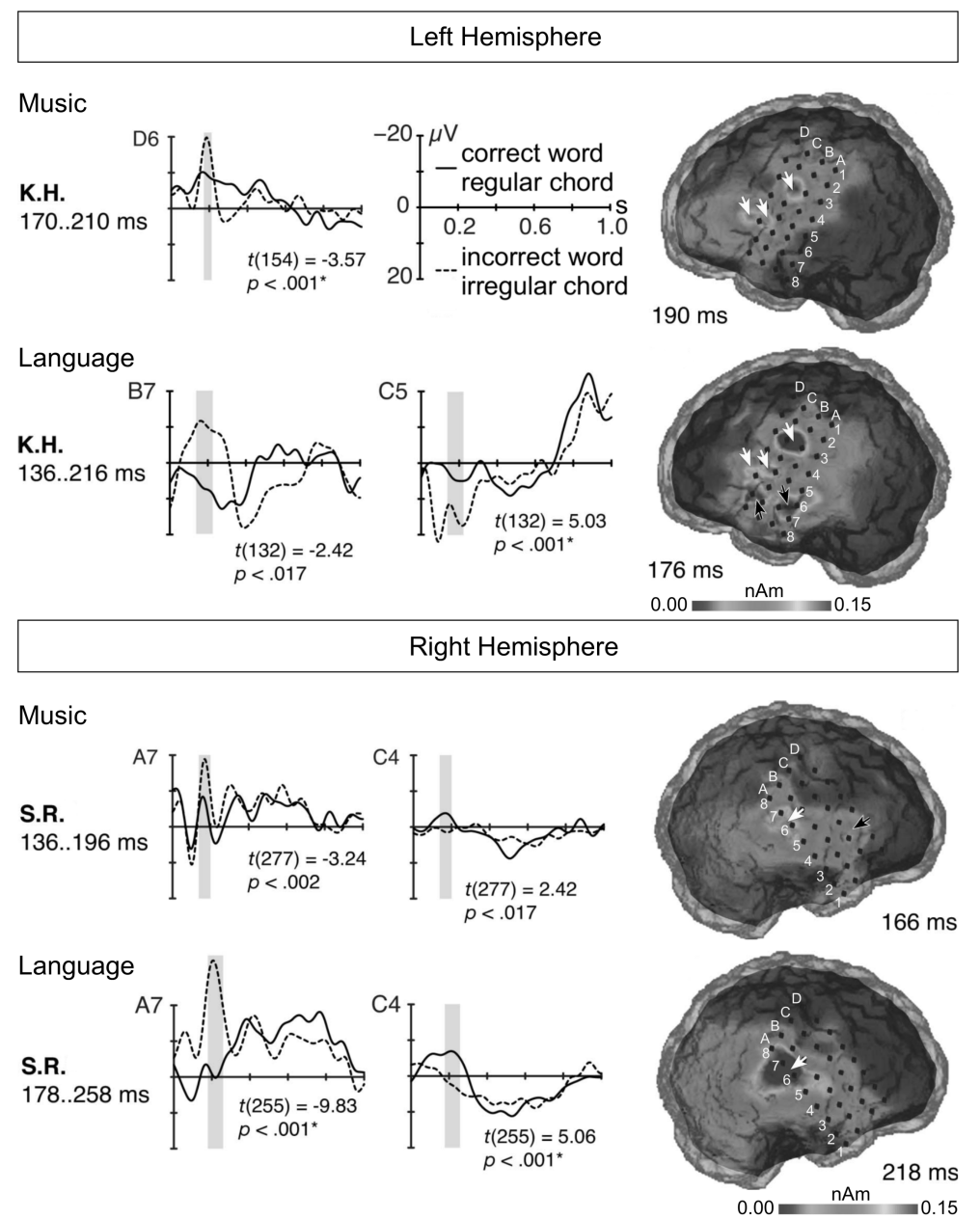

Figure 1. Music and language data (for the sake of brevity shown for only two representative patients): K.H. with left (upper panel) and S.R. with right perisylvian electrodes (lower panel). The ERP curves depict the most significant early negativity (left) and simultaneous early positivity (middle) in the music and the language experiment. The time window for statistical testing is shaded in gray and indicated next to the diagrams. Statistical values are depicted below each diagram; * indicates significance after Bonferroni correction. Results of the BSCD mapping (right) are plotted onto the individual, MNl-scaled brains. Black dots represent a reconstruction of the individual electrode positions. ${ }^{15}$ Electrode names are indicated as combinations of letters and numbers. White arrows point to generators observed in both domains, black arrows to sources detected in music or language only.

or right (three patients) perisylvian brain areas. Data analysis comprised the elimination of bad channels, downsampling to $500 \mathrm{~Hz}$, re-referencing to the common average reference of the grid, high- $(0.4 \mathrm{~Hz}, 6931$ points $)$ and low-pass (25 Hz, 213 points) filtering (fir), manual rejection of artifacts, and averaging within a 1000-ms poststimulus time window. Regular/correct and irregular/incorrect final chords/words were compared at each electrode by means of $t$-tests for independent samples (Bonferroni-corrected) within time windows centered around the peak of the greatest negativity occurring between 100 and $300 \mathrm{~ms}$ after onset of the critical element. Data were not averaged across patients on account of their variable grid positions (defined by medical needs). To localize the generators of the 
effects, brain surface current density (BSCD) mapping was applied on the peak of the difference waves (technical details are provided by Knösche et al. $\left.{ }^{11}\right)$.

\section{Results}

Syntactic violations in both language and music elicited early negativities irrespective of whether electrodes were implanted in the left or the right hemisphere (left row in Fig. 1), with an average peak latency of $193 \mathrm{~ms}$ in language (in all patients) and $188 \mathrm{~ms}$ in music (in five patients; four patients showed no significant effect, presumably because of low signal-to-noise-ratio or electrode position). Furthermore, simultaneous positivities were observed in both domains (middle row in Fig. 1). These patterns were consistent with the latency and the typical polarity inversion of the scalp-recorded ELAN ${ }^{3}$ and ERAN, ${ }^{4}$ suggesting that the observed effects represent the electrocortical equivalents of these surface ERP components.

The BSCD mapping identified generators within left and right superior temporal, inferior frontal, and inferior parietal brain areas in both the language and the music experiment (right panel of Fig. 1). Although the exact generator configurations varied between patients, considerable overlap of parts of these individual networks was observed when comparing the coordinates of music- and language-related effects within subjects. Areas of main overlap were located in the STG of both hemispheres. Furthermore, one patient (K.H.) showed clear overlap in the pars opercularis of the left IFG; however, further investigations with electrode grids centered on the frontal lobe are necessary to substantiate this finding.

\section{Conclusion}

Syntactic errors in language and in music elicited potentials around $200 \mathrm{~ms}$ that may be taken as the electrocortical equivalents of the
ELAN and the ERAN. The bilateral superior temporal and inferior frontal source localizations are consistent with previous studies, ${ }^{1,10-14}$ demonstrating the validity of the BSCD mapping when applied to intracranial ERPs.

The data confirm a co-localization of the early detection of musical and linguistic syntactic errors within the bilateral superior temporal and perhaps in the left inferior frontal lobe, as proposed by previous $\mathrm{MMRI}^{1,13,14}$ and MEG data. ${ }^{10-12}$ Overall, the present study adds (within-subject) anatomic evidence to theories of shared syntactic processing in music and language. ${ }^{1,2}$ Future studies could specify the overlap of these early syntactic processes with mechanisms of auditory oddball processing.

\section{Conflict of Interest}

The authors declare that they have no competing financial interests.

\section{References}

1. Koelsch, S. 2005. Neural substrates of processing syntax and semantics in music. Curr. Opin. Neurobiol. 15: $1-6$.

2. Patel, A.D. 2003. Language, music, syntax and the brain. Nat. Neurosci. 6: 674-681.

3. Friederici, A.D., E. Pfeifer \& A. Hahne. 1993. Eventrelated brain potentials during natural speech processing: effects of semantic, morphological and syntactic violations. Brain Res. Cogn. Brain Res. 1: 183192.

4. Koelsch, S., T. Gunter, A.D. Friederici, et al. 2000. Brain indices of music processing: "nonmusicians" are musical. f. Cogn. Neurosci. 12: 520-541.

5. Koelsch, S., T.C. Gunter, M. Wittfoth, et al. 2005. Interaction between syntax processing in language and music: an ERP study. F. Cogn. Neurosci. 17: 15651577.

6. Steinbeis, N. \& S. Koelsch. 2008. Shared neural resources between music and language indicate semantic processing of musical tension-resolution patterns. Cereb. Cortex 18: 1169-1178.

7. Jentschke, S. \& S. Koelsch. 2009. Musical training modulates the development of syntax processing in children. NeuroImage In press.

8. Jentsche, S., S. Koelsch, S. Sallat, et al. 2008. Children with specific language impairment also show 
impairment of music-syntactic processing. f. Cogn. Neurosci. 20: 1940-1951.

9. Patel, A.D., J.R. Iversen, M. Wassenaar, et al. 2008. Musical syntax processing in agrammatic Broca's aphasia. Aphasiology 22: 776-789.

10. Friederici, A.D., Y. Wang, C.S. Herrmann, et al. 2000. Localization of early syntactic processes in frontal and temporal cortical areas: a magnetoencephalographic study. Hum. Brain Mapp. 11: 111 .

11. Knösche, T.R., B. Maess \& A.D. Friederici. 1999. Processing of syntactic information monitored by brain surface current density mapping based on MEG. Brain Topogr. 12: 75-87.

12. Maess, B., S. Koelsch, T.C. Gunter, et al. 2001. Mu- sical syntax is processed in Broca's area: an MEG study. Nat. Neurosci. 4: 540-545.

13. Koelsch, S., T.C. Gunter, D.Y. Von Cramon, et al. 2002. Bach speaks: a cortical "language network" serves the processing of music. NeuroImage 17: 956966.

14. Friederici, A.D., S.A. Rüschemeyer, A. Hahne, et al. 2003. The role of left inferior frontal and superior temporal cortex in sentence comprehension: localizing syntactic and semantic processes. Cereb. Cortex 13: 170-177.

15. Kovalev, D., J. Spreer, J. Honegger, et al. 2005. Rapid and fully automated visualization of subdural electrodes in the presurgical evaluation of epilepsy patients. AJNR. Am. F. Neuroradiol. 26: 1078-1083. 